\title{
Expression and biological role of CIP2A in human astrocytoma
}

\author{
FUXIN YI, WEIMIN NI, WEIXIAN LIU, JINYANG BAI and WENBIN LI \\ Department of Neurosurgery, First Affiliated Hospital of Liaoning Medical University, \\ Jinzhou, Liaoning 121000, P.R. China
}

Received November 10, 2012; Accepted February 21, 2013

DOI: $10.3892 / \mathrm{mmr} .2013 .1357$

\begin{abstract}
Cancerous inhibitor of protein phosphatase 2A (CIP2A) is a recently characterized oncoprotein involved in the progression of several human malignancies. The present study aimed to investigate the clinical significance and biological function of CIP2A in astrocytoma. CIP2A expression was analyzed in 135 archived astrocytoma specimens using immunohistochemistry. Of these specimens, 75 cases (55.6\%) overexpressed CIP2A. The CIP2A overexpression was observed to be positively correlated with advanced tumor grade $(\mathrm{P}<0.001)$. siRNA-mediated knockdown of CIP2A was performed in A172 and U87 cell lines. MTT, colony formation and soft agar colony formation assays and Annexin V/propidium iodide analysis were performed to assess the role of CIP2A in cell proliferation and apoptosis. CIP2A depletion in the astrocytoma cell lines inhibited cell growth, reduced anchorage-independent cell growth and increased apoptosis. In addition, CIP2A depletion increased caspase-3 cleavage and downregulated c-Myc, Bcl-2 and phospho-Akt expression. These results validate the role of CIP2A as a clinically relevant oncoprotein and establish CIP2A as a promising therapeutic target of astrocytoma.
\end{abstract}

\section{Introduction}

Astrocytoma arises from neural stem or progenitor cells in the central nervous system and is the most common primary brain tumor, accounting for $\sim 60 \%$ of all brain tumors. Despite combined treatment strategies, including surgery, radiotherapy and chemotherapy, the prognosis for high-grade astrocytoma remains poor, with a median survival of $\sim 1$ year (1). The clinical symptoms and prognosis are closely correlated with tumor location, size and histological grade. Although the histological grade, in part, reflects the malignant features of astrocytoma, it is not able to provide an indication of the exact mechanism of tumor progression and recurrence. Thus, it is important

Correspondence to: Dr Fuxin Yi, Department of Neurosurgery, First Affiliated Hospital of Liaoning Medical University, 2 Renmin Street, Jinzhou 121000, P.R. China

E-mail: yifuxin@yahoo.com.cn

Key words: CIP2A, astrocytoma, proliferation, apoptosis to understand the molecular mechanism of astrocytoma cell progression and identify effective markers of tumorigenesis and progression.

Cancerous inhibitor of protein phosphatase 2A (CIP2A), originally named KIAA1524 or P90, has been cloned from hepatocellular carcinoma patients (2). CIP2A has been demonstrated to inhibit the activity of PP2A toward the oncogenic transcription factor, $\mathrm{c}-\mathrm{Myc}$, thereby preventing the proteolytic degradation of c-Myc, which is important for cell transformation and tumorigenesis in vivo and in vitro (3). In addition, CIP2A has been reported to reduce the apoptotic effect of bortezomib in breast cancer, hepatocellular carcinoma and head and neck squamous cell carcinoma (4-6). Moreover, CIP2A has been found to be overexpressed in various types of human cancer, including breast, gastric, lung, prostate, hepatocellular, ovarian, colon and renal cancers (6-17). The expression pattern of CIP2A in astrocytoma is not clear and the biological roles of CIP2A in astrocytoma cells remain to be examined.

In the present study, the expression pattern of CIP2A was investigated in 135 astrocytoma specimens and the correlations between CIP2A expression and clinicopathological factors were analyzed. Furthermore, to clarify the roles of CIP2A in astrocytoma, the effects of CIP2A on proliferation and apoptosis were investigated in astrocytoma cell lines.

\section{Materials and methods}

Patients and specimens. The study was approved by the institutional review board of Liaoning Medical University (Jinzhou, China). Primary tumor specimens were obtained from 135 patients diagnosed with astrocytoma who underwent resection in the First Affiliated Hospital of Liaoning Medical University between 2000 and 2005. The histological diagnosis was evaluated for sections stained with hematoxylin and eosin according to the World Health Organization classification guidelines. Clinical and histopathological data, including histopathological diagnosis and tumor grade, were extracted from medical records.

Cell culture and transfection. The U87 cell line was obtained from the American Type Culture Collection (Manassas, VA, USA) and the A172 cell line was from the Shanghai Cell Bank (Shanghai, China). Cells were cultured in Dulbecco's modified Eagle's medium (DMEM; Invitrogen Life Technologies, Carlsbad, CA, USA) containing $10 \%$ fetal bovine serum (FBS; Invitrogen Life Technologies), $100 \mathrm{IU} / \mathrm{ml}$ penicillin 
(Sigma-Aldrich, St. Louis, MO, USA) and $100 \mu \mathrm{g} / \mathrm{ml}$ streptomycin (Sigma-Aldrich). Cells were grown on sterilized culture dishes and were passaged every 2 days with $0.25 \%$ trypsin (Invitrogen Life Technologies).

On-TargetPlus SMARTpool CIP2A siRNA (\#L-014135-01) and On-TargetPlus siControl (D-001810-01-20) were purchased from Dharmacon (Thermo Fisher Scientific, Waltham, MA, USA). For transfections, cells were seeded in plates $24 \mathrm{~h}$ prior to the experiment. Cells were transfected with siRNA using DharmaFECT 1 (0.20 $\mu \mathrm{l} /$ well; Thermo Fisher Scientific) according to the manufacturer's instructions.

Immunohistochemistry. Sections (4- $\mu \mathrm{m}$ thick) were prepared from paraffin-embedded tissues. Immunostaining was performed by the streptavidin-peroxidase method (Ultrasensitive; MaiXin, Fuzhou, China). Sections were deparaffinized in xylene, rehydrated with graded alcohol and then boiled in citrate buffer ( $\mathrm{pH}$ 6.0) for $2 \mathrm{~min}$ in an autoclave. Next, $0.3 \%$ hydrogen peroxide was applied to block endogenous peroxidase activity and the sections were incubated with normal animal serum to reduce nonspecific binding. Tissue sections were incubated with CIP2A rabbit polyclonal antibody (1:300 dilution; Novus Biologicals, LCC, Littleton, CO, USA) for $2 \mathrm{~h}$ at room temperature. Rabbit immunoglobulin (at the same concentration as the antigen-specific antibody) was used as a negative control. Staining was followed by incubation with biotinylated secondary antibodies. The peroxidase reaction was developed with 3,3'-diaminobenzidine tetrahydrochloride. Counterstaining was performed lightly with hematoxylin and the sections were dehydrated in alcohol prior to mounting.

Immunostaining of CIP2A was scored on a semiquantitative scale by evaluating the intensity and percentage of tumor cells as described previously (10). We counted 400 tumor cells and calculated the percentage of positively stained cells. The intensity of CIP2A staining was scored as 0 (no signal), 1 (weak), 2 (moderate) and 3 (marked). Percentage scores were assigned as $1,1-25 \% ; 2,26-50 \% ; 3,51-75 \%$; and 4, 76-100\%. The scores of each tumor sample were multiplied to produce a final score of $0-12$ and the tumors were finally determined as negative $(-), 0$; lower expression $(+), \leq 4$; moderate expression $(++), 5-8$; and high expression $(+++), \geq 9$. Tumor samples that scored $(+)$ to $(+++)$ were considered to overexpress CIP2A.

Quantitative real-time PCR. Quantitative real-time PCR was performed using the SYBR-Green PCR master mix in a total volume of $20 \mu \mathrm{l}$ on a 7900HT Fast Real-Time PCR System (both Applied Biosystems, Bedford, MA, USA) as follows: $95^{\circ} \mathrm{C}$ for $30 \mathrm{sec}$ and 40 cycles at $95^{\circ} \mathrm{C}$ for $5 \mathrm{sec}$ and $60^{\circ} \mathrm{C}$ for $30 \mathrm{sec}$. A dissociation step was performed to generate a melting curve to confirm the specificity of the amplification. Expression levels of the analyzed genes were normalized against $\beta$-actin expression.Relative levels of gene expression were determined using the following formula: $\Delta \mathrm{Ct}=\mathrm{Ct}_{\mathrm{gene}}-\mathrm{Ct}_{\mathrm{ref}}$ and the fold change of gene expression was calculated by the $2^{-\Delta \Delta \mathrm{Ct}}$ method. The primer sequences were as follows: CIP2A, 5'-ATACTTCAGGACCCACGTTTGAT-3' (forward) and 5'-TCTCCAAGTACTAAAGCAGGA AAATCT-3' (reverse); $\beta$-actin, 5'-ATAGCACAGCCTGGA TAGCAACGTAC-3' (forward) and 5'-CACCTT
CTACAATGAGCTGCGTGTG-3' (reverse). Experiments were repeated in triplicate.

Western blot analysis. Total protein from tissue and cells was extracted in lysis buffer (Pierce Biotechnology, Inc., Rockford, IL, USA) and quantified using the Bradford method. Samples were separated by SDS-PAGE and transferred to polyvinylidene fluoride membranes (Millipore, Billerica, MA, USA) and incubated overnight at $4^{\circ} \mathrm{C}$ with antibodies against CIP2A (1:1,000; Novus Biologicals, LCC), caspase-3, cleaved caspase-3, c-Myc, phospho-Akt, Akt, Bcl-2 (1:800, Cell Signaling Technology, Inc., Danvers, MA, USA) and $\beta$-actin (1:500; Santa Cruz Biotechnology, Inc., Santa Cruz, CA, USA). Following incubation with peroxidase-coupled anti-mouse IgG (Santa Cruz Biotechnology, Inc.) at $37^{\circ} \mathrm{C}$ for $2 \mathrm{~h}$, bound proteins were visualized using an ECL detection system (Pierce Biotechnology, Inc.) and detected using a BioImaging System (UVP Inc., Upland, CA, USA). Relative protein levels were quantified using $\beta$-actin as a loading control.

Colony formation, anchorage-independent colony formation and thiazolyl blue (MTT) assays. For the colony formation assay, A172 and U87 cells were transfected with siRNA for $48 \mathrm{~h}$ and plated into three $6-\mathrm{cm}$ cell culture dishes (1,000 cell/dish). Cells were incubated for 12 days in medium containing $10 \%$ FBS. The plates were washed with phosphate-buffered saline (PBS) and stained with Giemsa. The number of colonies with $>50$ cells was counted manually using a microscope.

For the anchorage-independent colony growth assay $\sim 2,000$ cells/well were seeded in medium containing $0.5 \%$ agarose on top of bottom agar containing $1 \%$ low-melting agar in regular medium. After 14-21 days, colonies were stained with Giemsa and counted using a microscope.

For the MTT assay, cells were plated in 96-well plates in medium containing 10\% FBS at $~ 3,000$ cells/well $24 \mathrm{~h}$ following transfection. For quantification of cell viability, $20 \mu \mathrm{l}$ MTT $(5 \mathrm{mg} / \mathrm{ml})$ solution was added to each well and incubated for $4 \mathrm{~h}$ at $37^{\circ} \mathrm{C}$. The medium was removed from each well and the resulting MTT formazan was solubilized in $150 \mu \mathrm{l}$ DMSO. Each solution was measured spectrophotometrically at $490 \mathrm{~nm}$.

Apoptosis analysis. Cells $\left(5 \times 10^{5}\right)$ were seeded into $6-\mathrm{cm}$ tissue culture dishes. After $12 \mathrm{~h}$, the cells were transfected with siRNA using DharmaFECT $1(0.20 \mu \mathrm{l} /$ well $)$. For the detection of apoptosis, adherent cells were collected and resuspended in cold PBS for analysis. The cells were stained with the Annexin V-FITC Apoptosis kit (BD Pharmingen, San Diego, CA, USA) to monitor apoptotic cells and propidium iodide (PI) to detect dead cells. Data were collected using a BD FACSCalibur flow cytometer (San Jose, CA, USA).

Statistical analysis. SPSS version 11.5 for Windows was used for all statistical analyses (SPSS, Inc., Chicago, IL, USA). A $\chi^{2}$ test was used to examine the possible correlations between CIP2A expression and clinicopathological factors. The Student's t-test was used to compare densitometry data on focus numbers between control and CIP2A-transfected cells. All P-values are based on a two-sided statistical analysis and $\mathrm{P}<0.05$ was considered to indicate a statistically significant difference. 
Table I. Relationship between cancerous inhibitor of protein phosphatase 2A (CIP2A) and clinicopathological features

\begin{tabular}{lrrrr}
\hline & \multicolumn{3}{c}{ CIP2A } \\
\cline { 3 - 4 } $\begin{array}{l}\text { Clinical } \\
\text { parameters }\end{array}$ & $\mathrm{n}$ & Negative & Positive & P-value \\
\hline Age & & & & \\
$\quad<45$ years & 67 & 33 & 34 & 0.301 \\
$\geq 45$ years & 68 & 27 & 41 & \\
Gender & & & & \\
Male & 82 & 37 & 45 & 0.861 \\
Female & 53 & 23 & 30 & \\
Grading & & & & \\
I & 9 & 8 & 1 & $<0.001$ \\
II & 66 & 36 & 31 & \\
III & 42 & 10 & 32 & \\
IV & 18 & 7 & 11 & \\
\hline
\end{tabular}
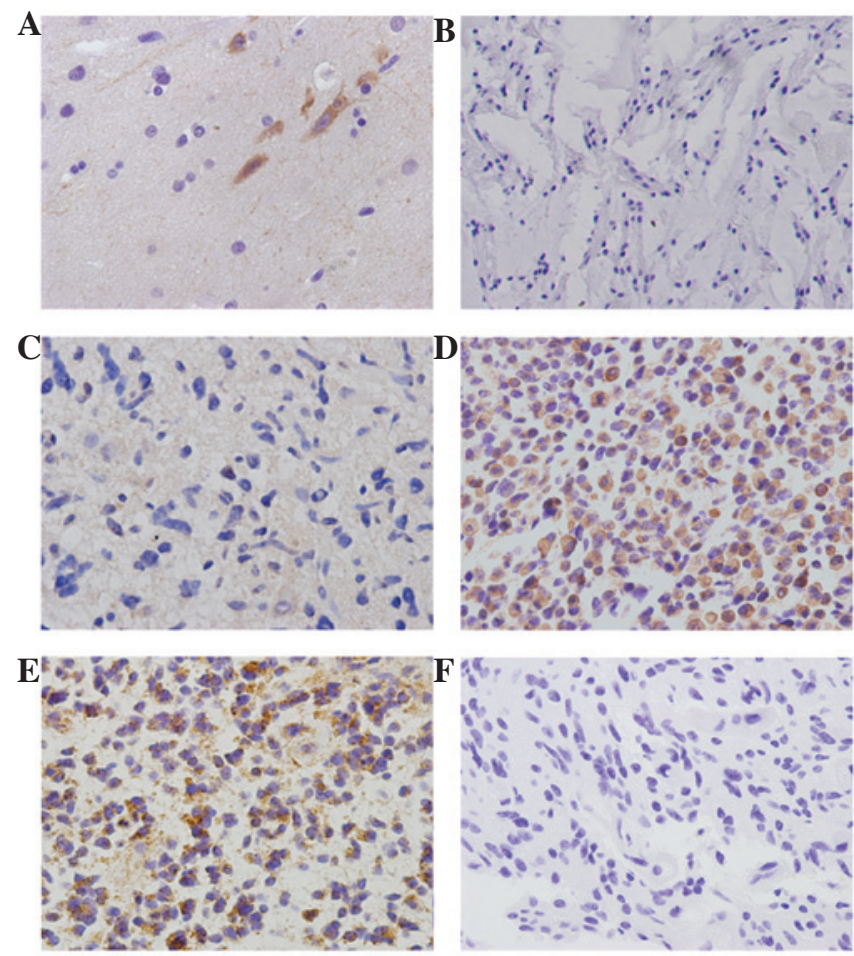

Figure 1. Expression of CIP2A in resected astrocytoma tissue. (A) Immunohistochemical staining of CIP2A protein in normal brain tissue was negative in glial cells and positive in neurons. (B) Negative CIP2A staining in pilocytic astrocytoma (grade I). (C) Negative CIP2A staining in grade II glioma. (D) Positive cytoplasmic CIP2A staining in grade III astrocytoma. (E) Positive cytoplasmic CIP2A staining in glioblastoma (grade IV). (F) Negative control. Magnification, x200. CIP2A, cancerous inhibitor of protein phosphatase $2 \mathrm{~A}$.

\section{Results}

Expression of CIP2A in astrocytoma. In normal brain tissues, negative expression of CIP2A in astrocytes and positive cytoplasmic CIP2A expression in neurons was observed (Fig. 1A). Positive cytoplasmic CIP2A staining was observed in 75/135
A
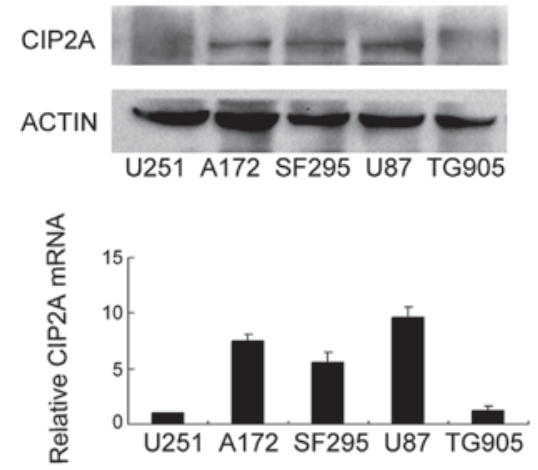

B
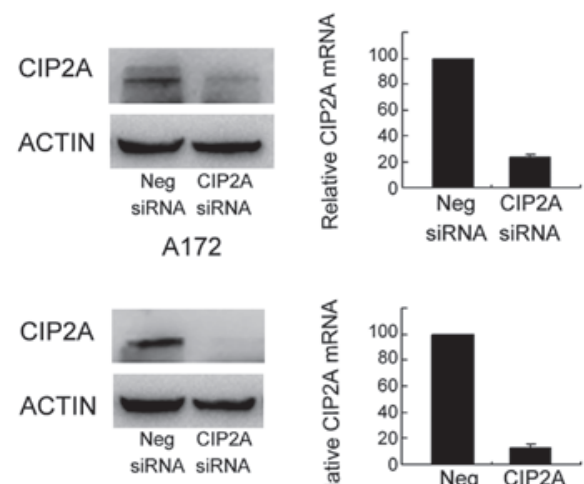

U87

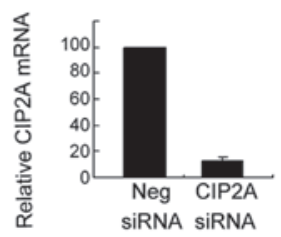

Figure 2. CIP2A expression and knockdown efficiency in astrocytoma cell lines. (A) Endogenous expression of CIP2A was examined in five astrocytoma cell lines by western blot analysis and real-time PCR. (B) western blot analysis and real-time PCR revealed that siRNA treatment of CIP2A markedly decreased CIP2A levels in A172 and U87 cells compared with negative control cells. CIP2A, cancerous inhibitor of protein phosphatase $2 \mathrm{~A}$.

$(55.6 \%)$ human astrocytomas (Fig. 1B-E), while no staining was detected in sections from the same samples subjected to immunohistochemical analysis using non-immune rabbit immunoglobulin (Fig. 1F). The correlations between CIP2A protein expression and clinicopathological factors was investigated and no relationship was found between CIP2A expression and age and gender. The positive rates of CIP2A overexpression in grades I (Fig. 1B), II (Fig. 1C) and III astrocytomas (Fig. 1D) and grade IV astrocytoma/glioblastoma (Fig. 1E) were 11.1 (1/9), 47.0 (31/66), 76.2 (32/42) and 61.1\% (11/18), respectively (Table I). The positive rate of CIP2A was identified to be significantly higher in high-grade astrocytomas than in those of low-grade $(\mathrm{P}<0.001)$.

CIP2A depletion in astrocytoma cell lines inhibits cell proliferation and increases cell apoptosis. To determine whether CIP2A enhances the proliferation of astrocytoma, CIP2A expression levels were analyzed in several astrocytoma cell lines and A172 and U87 cells were found to exhibit high levels of CIP2A expression (Fig. 2A). siRNA-mediated knockdown of CIP2A was performed in these cell lines. As demonstrated in Fig. 2B, CIP2A siRNA decreased the levels of CIP2A protein and mRNA in the A172 and U87 cells. The proliferation rate of the cells was determined by MTT assay. A172 and U87 cells treated with CIP2A siRNA exhibited a significantly slower growth rate than the vector control cells (Fig. 3A). Consistent with MTT results, the colony formation assay 
A
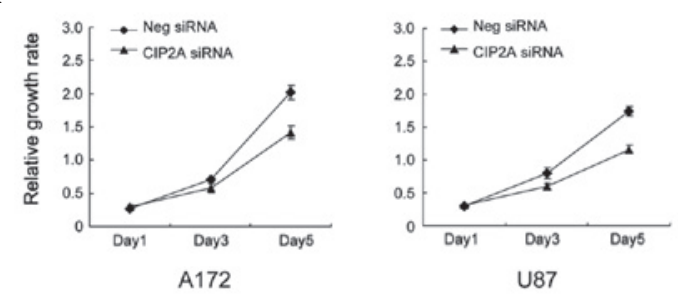

B

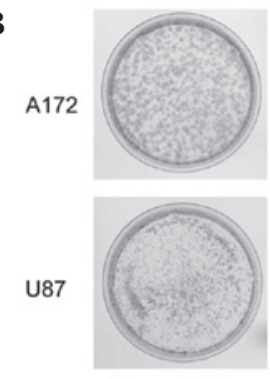

Neg siRNA

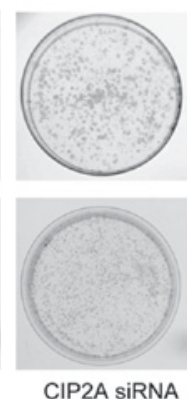

CIP2A SiRNA
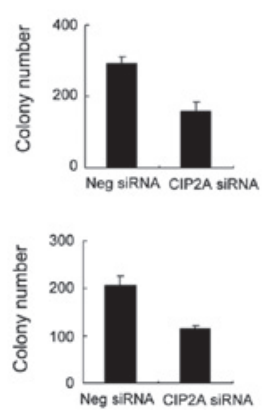

C A172
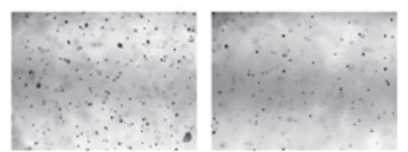

U87

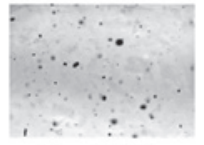

Neg siRNA

D

A172

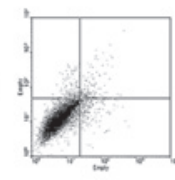

U87

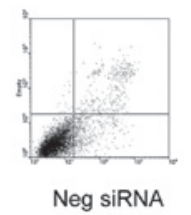

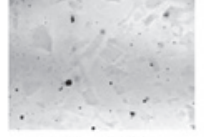

CIP2A siRNA
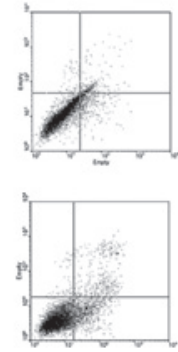

CIP2A siRNA
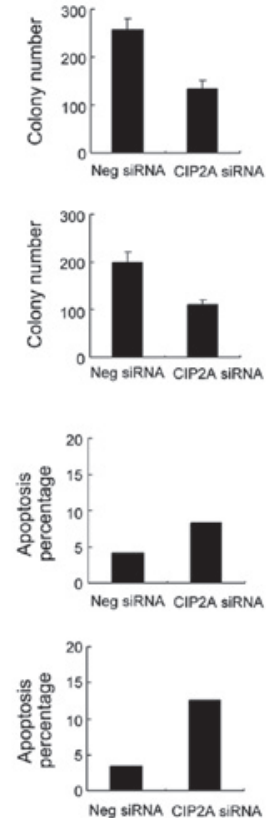

Figure 3 CIP2A knockdown inhibited cell proliferation in astrocytoma cell lines. (A) MTT assay revealed that CIP2A depletion inhibited cell proliferation. (B) Assessment of the clonogenic potential of CIP2A depleted glioma cells. The number of colonies formed by cells treated with CIP2A siRNA was reduced compared with control $(\mathrm{P}<0.05)$. (C) Anchorage-independent colony formation assay revealed that CIP2A knockdown inhibited glioma cell growth in soft agar $(\mathrm{P}<0.05)$. (D) Annexin V/PI analysis revealed that CIP2A knockdown increased the level of apoptosis in astrocytoma cell lines. CIP2A, cancerous inhibitor of protein phosphatase $2 \mathrm{~A}$.

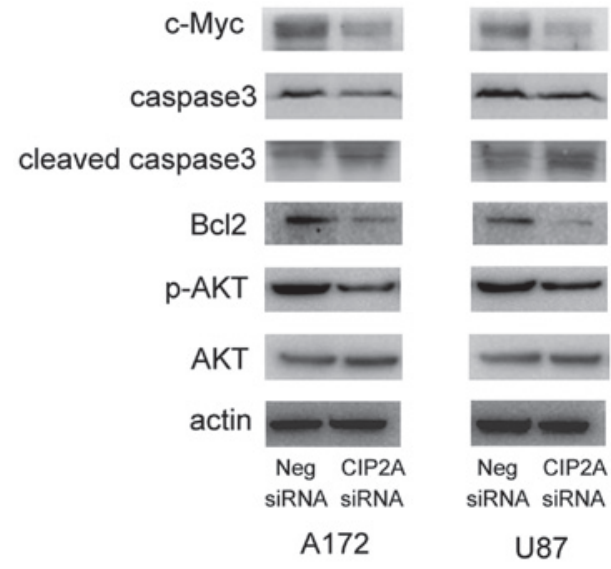

Figure 4. CIP2A siRNA treatment downregulated c-Myc, Bcl-2, phosphor-Akt expression and increased caspase- 3 cleavage in the astrocytoma cell line. Western blot analysis revealed that siRNA knockdown of CIP2A decreased the protein levels of c-Myc, Bcl-2 and phospho-Akt in the two cell lines. Levels of cleaved caspase-3 were increased following CIP2A depletion. CIP2A, cancerous inhibitor of protein phosphatase 2A.

revealed that CIP2A-knockdown in A172 and U87 cells led to a marked decrease in focus numbers (A172: control 292 \pm 17 vs. CIP2A siRNA $157 \pm 26, \mathrm{P}<0.05$; U87: control 207 \pm 19 vs. CIP2A siRNA $119 \pm 6, \mathrm{P}<0.05$; Fig. 3B). To examine the impact of CIP2A on anchorage-independent cell growth, a soft agar colony formation assay was performed. CIP2A-knockdown reduced the colony numbers in soft agar (A172: control 257 \pm 22 vs. CIP2A siRNA $133 \pm 16, \mathrm{P}<0.05$; U87: control 199 \pm 21 vs. CIP2A siRNA $109 \pm 11, \mathrm{P}<0.05$ )

Annexin V/PI analysis was employed to characterize the rate of apoptosis. As demonstrated in Fig. 3D, the populations of cells with CIP2A-knockdown that were observed to be undergoing early and late apoptosis (A172: 8.7\%; U87: 13.2\%) were significantly larger compared with scramble controls (A172: 4.6\%; U87: $3.9 \%$ ), demonstrating that CIP2A-knockdown results in the apoptosis of astrocytoma cells.

CIP2A depletion increases caspase-3 cleavage, downregulates $\mathrm{c}-\mathrm{Myc}$ and $\mathrm{Bcl}-2$ expression and inhibits Akt phosphorylation in astrocytoma cells. To investigate the underlying mechanism by which CIP2A affects proliferation and apoptosis, the effect of CIP2A-knockdown on several potential molecular targets was analyzed. As revealed in Fig. 4, western blot analysis demonstrated that the knockdown of CIP2A decreased the expression levels of c-Myc protein in the two cell lines. In addition, the levels of apoptosis-related caspase-3 and Bcl-2 expression were determined and it was observed that CIP2A depletion led to reductions in caspase-3 and $\mathrm{Bcl}-2$ protein levels and increased the levels of cleaved caspase-3. In addition, Akt phosphorylation levels were markedly lower in the cells treated with CIP2A siRNA. Together, these results indicate that $\mathrm{CIP} 2 \mathrm{~A}$ regulates cell proliferation and apoptosis through modulation of $\mathrm{c}-\mathrm{Myc}, \mathrm{Bcl}-2$ and $\mathrm{Akt}$ levels.

\section{Discussion}

The present study demonstrated that CIP2A was overexpressed in $55.6 \%$ of human astrocytoma and correlated with increased tumor grade. In addition, CIP2A depletion in two astrocytoma cell lines was demonstrated to inhibit cell growth and anchorage-independent cell growth and increase apoptosis, by downregulating c-Myc, Bcl-2 and phospho-Akt protein and upregulating caspase-3 cleavage. 
CIP2A has been reported to be overexpressed in various types of cancer, including breast, gastric, lung, prostate, hepatocellular, colon and renal cancers $(4,6-10,14,17)$. A previous study reported that the overexpression of CIP2A increases and siRNA silencing of CIP2A decreases the self-renewal and proliferation of mouse neural progenitor cells (18). However, the expression patterns and biological roles of CIP2A in human astrocytoma remain largely unknown. In the current study, marked cytoplasmic CIP2A expression was found in neurons and negative staining was identified in human glial cells, including astrocytes. CIP2A overexpression was found in $55.6 \%$ of the astrocytoma tissues examined. In addition, a close association between CIP2A overexpression and the astrocytoma grade was identified.

Different gene expression patterns have been proposed in the development of low- vs. high-grade astrocytomas, which reflects the malignant potential of high invasive capability and growth rate (19). The higher expression rate of CIP2A in high-grade (III-IV) compared with low-grade carcinomas indicates its potential association with the aggressiveness of astrocytoma cells.

To determine the role of CIP2A in astrocytoma cells, CIP2A expression was knocked down in A172 and U87 cell lines. Consistent with previous studies, CIP2A depletion was found to significantly decrease the proliferation rate, colony formation ability and anchorage-independent growth of A172 and U87 cell lines. To examine the potential mechanism, the effect of CIP2A-knockdown on c-Myc expression, a target protein of CIP2A, was examined. CIP2A depletion markedly downregulated c-Myc expression. c-Myc is a cellular proto-oncogene associated with a variety of types of human cancer and is associated with control of cellular proliferation (20). Loss of c-Myc is associated with reduction of cyclin D1-Cdk4 and cyclin D1-Cdk6 complexes during the cell cycle transition (21). Overexpression of CIP2A has been demonstrated to upregulate Akt and protect cells from bortezomib-induced apoptosis in hepatocellular and head and neck squamous cell carcinoma cells $(4,5)$. In the present study, CIP2A-knockdown was observed to facilitate the apoptosis of astrocytoma cells.

Caspase-3 is a critical executioner of apoptosis. Activation of caspase- 3 requires proteolytic processing of its inactive zymogen into activated p17 and p12 fragments (22). In the current study, CIP2A depletion increased caspase-3 cleavage, consistent with increased apoptosis.

$\mathrm{Bcl}-2$ prevents cells from undergoing apoptosis in response to a variety of stimuli and is hypothesized to be involved in resistance to conventional cancer treatment. It has been reported that activation of the Akt pathway modulates Bcl-2 expression. In the present study, CIP2A depletion reduced Bcl-2 protein levels and Akt phosphorylation (23). These results indicate that CIP2A regulates cell apoptosis via $\mathrm{Bcl}-2$ and Akt activation.

CIP2A is overexpressed in astrocytomas and correlates with tumor grade. CIP2A depletion attenuates cell proliferation and facilitates apoptosis. In addition, CIP2A depletion increases caspase-3 cleavage and inhibits c-Myc, Bcl-2 and phospho-Akt expression. These results are likely to provide insight into the functional importance of CIP2A in the progression of human astrocytoma. The observations of the present study indicate that CIP2A represents a molecular target closely associated with cell proliferation and apoptosis and may provide a basis for the future development of cancer therapeutics.

\section{References}

1. Bondy ML, Scheurer ME, Malmer B, et al: Brain tumor epidemiology: consensus from the Brain Tumor Epidemiology Consortium. Cancer 113: 1953-1968, 2008.

2. Soo Hoo L, Zhang JY and Chan EK: Cloning and characterization of a novel $90 \mathrm{kDa}$ 'companion' auto-antigen of p62 overexpressed in cancer. Oncogene 21: 5006-5015, 2002.

3. Junttila MR, Puustinen P, Niemela M, et al: CIP2A inhibits PP2A in human malignancies. Cell 130: 51-62, 2007.

4. Chen KF, Liu CY, Lin YC, et al: CIP2A mediates effects of bortezomib on phospho-Akt and apoptosis in hepatocellular carcinoma cells. Oncogene 29: 6257-6266, 2010.

5. Lin YC, Chen KC, Chen CC, Cheng AL and Chen KF: CIP2A-mediated Akt activation plays a role in bortezomib-induced apoptosis in head and neck squamous cell carcinoma cells. Oral Oncol 48: 585-593, 2012.

6. Come C, Laine A, Chanrion M, et al: CIP2A is associated with human breast cancer aggressivity. Clin Cancer Res 15: 5092-5100, 2009.

7. Basile JR and Czerninski R: The role of CIP2A in oral squamous cell carcinoma. Cancer Biol Ther 10: 700-702, 2010.

8. Bockelman C, Koskensalo S, Hagstrom J, Lundin M, Ristimaki A and Haglund C: CIP2A overexpression is associated with c-Myc expression in colorectal cancer. Cancer Biol Ther 13: 289-295, 2012.

9. Bockelman C, Lassus H, Hemmes A, et al: Prognostic role of CIP2A expression in serous ovarian cancer. Br J Cancer 105: 989-995, 2011.

10. Dong QZ, Wang Y, Dong XJ, et al: CIP2A is overexpressed in non-small cell lung cancer and correlates with poor prognosis. Ann Surg Oncol 18: 857-865, 2011.

11. Huang LP, Adelson ME, Mordechai E and Trama JP: CIP2A expression is elevated in cervical cancer. Cancer Biomark 8: 309-317, 2010-2011.

12. Katz J, Jakymiw A, Ducksworth MK, et al: CIP2A expression and localization in oral carcinoma and dysplasia. Cancer Biol Ther 10: 694-699, 2010

13. Qu W, Li W, Wei L, Xing L, Wang X and Yu J: CIP2A is overexpressed in esophageal squamous cell carcinoma. Med Oncol 29: 113-118, 2012.

14. Ren J, Li W, Yan L, et al: Expression of CIP2A in renal cell carcinomas correlates with tumour invasion, metastasis and patients' survival. Br J Cancer 105: 1905-1911, 2011.

15. Teng HW, Yang SH, Lin JK, et al: CIP2A is a predictor of poor prognosis in colon cancer. J Gastrointest Surg 16: 1037-1047, 2012.

16. Vaarala MH, Vaisanen MR and Ristimaki A: CIP2A expression is increased in prostate cancer. J Exp Clin Cancer Res 29: 136, 2010.

17. Li W, Ge Z, Liu C, et al: CIP2A is overexpressed in gastric cancer and its depletion leads to impaired clonogenicity, senescence, or differentiation of tumor cells. Clin Cancer Res 14: 3722-3728, 2008.

18. Kerosuo L, Fox H, Perala N, et al: CIP2A increases self-renewal and is linked to Myc in neural progenitor cells. Differentiation 80: 68-77, 2010.

19. Chow LM, Endersby R, Zhu X, et al: Cooperativity within and among Pten, p53 and Rb pathways induces high-grade astrocytoma in adult brain. Cancer Cell 19: 305-316, 2011.

20. Gordan JD, Thompson CB and Simon MC: HIF and c-Myc: sibling rivals for control of cancer cell metabolism and proliferation. Cancer Cell 12: 108-113, 2007.

21. Mateyak MK, Obaya AJ and Sedivy JM: c-Myc regulates cyclin D-Cdk4 and -Cdk6 activity but affects cell cycle progression at multiple independent points. Mol Cell Biol 19: 4672-4683, 1999.

22. D'Amelio M, Cavallucci V and Cecconi F: Neuronal caspase-3 signaling: not only cell death. Cell Death Differ 17: 1104-1114, 2010.

23. Pugazhenthi S, Nesterova A, Sable C, et al: Akt/protein kinase $\mathrm{B}$ up-regulates Bcl-2 expression through cAMP-response element-binding protein. J Biol Chem 275: 10761-10766, 2000. 